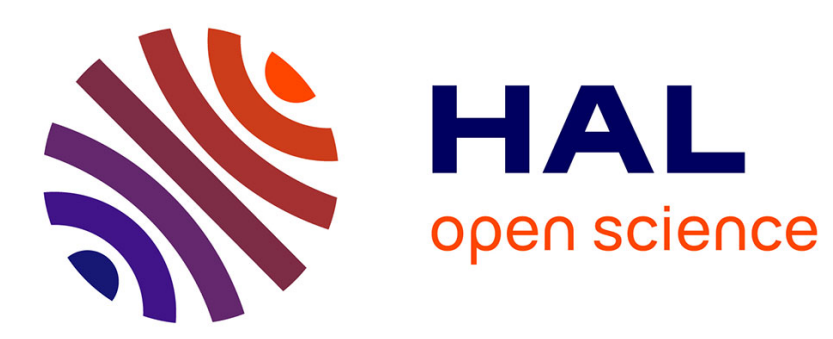

\title{
Déterminisme des choix alimentaires des herbivores au pâturage: principales théories
}

\author{
Bertrand Dumont
}

\section{To cite this version:}

Bertrand Dumont. Déterminisme des choix alimentaires des herbivores au pâturage: principales théories. Productions Animales, 1995, 8 (4), pp.285-292. hal-00896125

\section{HAL Id: hal-00896125 \\ https://hal.science/hal-00896125}

Submitted on 1 Jan 1995

HAL is a multi-disciplinary open access archive for the deposit and dissemination of scientific research documents, whether they are published or not. The documents may come from teaching and research institutions in France or abroad, or from public or private research centers.
L'archive ouverte pluridisciplinaire HAL, est destinée au dépôt et à la diffusion de documents scientifiques de niveau recherche, publiés ou non, émanant des établissements d'enseignement et de recherche français ou étrangers, des laboratoires publics ou privés. 
INRA Prod. Anim., 1995, 8 (4), 285-292

\section{B. DUMONT}

INRA Adaptation des Herbivores aux Milieux

Theix 63122 Saint-Genès-Champanelle
Déterminisme des choix alimentaires des herbivores au pâturage : principales théories

Comment mieux gérer les zones agricoles en déprise ? Comment les herbivores peuvent-ils contribuer à entretenir ces espaces ? Comment utiliser au mieux les choix alimentaires des animaux rendus à une certaine liberté de consommation dans un milieu hétérogène ? Ces questions d'actualité en France expliquent l'importance croissante des recherches conduites à l'INRA sur le pâturage extensif. Cet article présente les principales théories visant à expliquer les choix alimentaires des herbivores au pâturage.

La demande socio-économique pour la gestion de l'espace rural est devenue pressante. Les herbivores peuvent contribuer à y répondre, en jouant en particulier un rôle direct dans la maîtrise de la végétation par le pâturage. Aujourd'hui, dans certaines zones d'élevage, l'équilibre des ressources productives, écologiques et paysagères, que maintenaient jusqu'alors les éleveurs et leurs troupeaux, est d'ores et déjà menacé par une certaine désertification. Celle-ci entraîne une sous-exploitation des ressources fourragères qui conduit à plus ou moins long terme à un

\footnotetext{
Résumé

L'objectif d'entretien d'espaces par le pâturage nécessite de mieux comprendre les règles qui président aux choix alimentaires des herbivores. Les études dans ce domaine se sont longtemps articulées autour de l'Optimal Foraging Theory, qui postule que les décisions des animaux sont dictées par la volonté de maximiser leur bilan énergétique. Cependant, afin de mieux rendre compte de la diversité des régimes observés, d'autres approches ont été proposées, inspirées pour une large part par les contraintes nutritionnelles que subissent les herbivores. II semble clair que les caractéristiques morpho-physiologiques des animaux expliquent largement les différences de choix entre espèces. En revanche, au sein de chaque espèce, les expériences alimentaires dans le jeune âge, l'apprentissage avec la mère et la reconnaissance des conséquences post-ingestives des choix réalisés jouent un rôle dans l'acquisition d'habitudes alimentaires et expliquent à la fois une certaine "sagesse nutritionnelle " et la plasticité des comportements observés.
}

envahissement de l'espace par une végétation indésirable. Cette évolution se traduit par l'embroussaillement des parcours, qui a comme conséquence la plus spectaculaire, en zone méditerranéenne, le développement des incendies. Cependant, des zones herbagères plus productives sont elles aussi concernées. L'un des enjeux est alors de savoir gérer ces surfaces de façon extensive dans des systèmes d'élevage qui feront le moins possible appel aux interventions humaines (fauche,...) et où le pâturage aura un rôle prédominant.

Ces systèmes de conduite des troupeaux et des surfaces devront aboutir à de nouveaux équilibres acceptables pour tous. Ils concernent en priorité les troupeaux allaitants bovins et ovins, les plus nombreux dans les zones herbagères difficiles et menacées, mais qui se développent aussi dans des régions plus riches où le nombre de vaches laitières diminue. Ces troupeaux devront contribuer à entretenir l'espace, mais évidemment également assurer une production qui permettra par ailleurs de réduire les coûts de cet entretien.

Par ses choix alimentaires, l'herbivore a un impact déterminant sur l'évolution de la flore, de l'environnement et des ressources fourragères, pouvant favoriser ou, à l'inverse, surexploiter certains éléments du couvert. L'objectif d'entretien d'espaces par le pâturage nécessite donc de mieux comprendre les 
règles qui président aux choix alimentaires des différentes espèces d'herbivores que l'on souhaite utiliser.

\section{1 / L'Optimal Foraging Theory}

Les études sur les choix alimentaires des herbivores se sont longtemps articulées autour de la théorie de l'alimentation optimale (ou Optimal Foraging Theory : Schœener 1971, Pyke et al 1977, Stephens et Krebs 1986), qui postule que les décisions des animaux sont dictées par la "volonté " de maximiser leur bilan énergétique. Si cette théorie permet de prévoir avec succès la sélection alimentaire des carnivores ou des nectarivores, les contraintes particulières auxquelles sont confrontés les herbivores (ressources aisément disponibles mais de qualité hétérogène et variable dans le temps, régulations particulières des quantités ingérées chez les ruminants) ont nécessité des adaptations du modèle général. Elles ont été proposées dans le sens d'une optimisation de l'ingestion de rations équilibrées (Westoby 1974), ou au moins en tenant compte de la nécessité que l'herbivore ingère une quantité minimale de certains nutriments (Belovsky 1978, OwenSmith et Novellie 1982). Ces modèles tiennent également compte des contraintes digestives, particulièrement dans plusieurs travaux récents (Verlinden et Wiley 1989, Owen-Smith 1993, Newman et al 1995). Les modèles qui s'inspirent de l'Optimal Foraging Theory s'expriment selon différents types de formulation, les deux principales étant les modèles de programmation linéaire (Westoby 1974, Belovsky 1978) et les modèles de contingence (Owen-Smith et Novellie 1982, Verlinden et Wiley 1989).

S'alimenter de manière optimale peut réduire la durée d'ingestion journalière, périodes au cours desquelles les herbivores sont le plus sensible à la prédation. Cela permet aussi aux animaux de pâturer en dehors des heures où les contraintes thermiques sont les plus fortes, et de disposer de plus de temps pour d'autres activités (reproduction, soins aux jeunes,...). Un animal pourrait s'alimenter d'une manière optimale tout en satisfaisant à certaines contraintes nutritionnelles sans nécessairement percevoir la valeur de ce qu'il ingère. Pour cela, il suffit qu'il oriente ses choix à partir de certaines caractéristiques des organes végétaux consommés (Cassini 1994). Il a, en effet, de grandes chances de satisfaire ses besoins en suivant quelques règles simples comme celles de sélectionner des jeunes pousses en croissance ou les placettes d'herbe les plus hautes et les plus vertes.

Même si les modèles qui s'inspirent de l'Optimal Foraging ont permis de prévoir avec plus ou moins de succès le régime de différentes espèces d'herbivores dans des situations concrètes de pâturage (e.g. Belovsky 1978, 1986, Owen-Smith et Novellie 1982, Owen-Smith 1993), il ne semble pas que les herbivores s'alimentent systématiquement d'une manière optimale. Par exemple, alors que les moutons choisissent parfois, à l'auge (Kenney et Black 1984) ou au pâturage (Arnold 1987), les fourrages qui permettent l'ingestion la plus rapide, ils peuvent en revanche être très sélectifs, bien que cela limite leur vitesse d'ingestion, sur des prairies où la quantité d'herbe est pourtant limitante (Broom et Arnold 1986). De plus, quand ils ont le choix entre du trèfle et du ray-grass, les moutons sélectionnent les deux espèces (Parsons et al 1994) bien que leur vitesse d'ingestion soit plus élevée sur le trèfle (Penning et al 1991). Dans ce dernier exemple, la question est d'ailleurs relancée depuis que Newman et al (1995) ont calculé qu'un régime mixte représenterait le meilleur compromis entre vitesses d'ingestion, transit et absorption des nutriments. Plus généralement, une limite à l'application de l'Optimal Foraging Theory aux herbivores est que les différents modèles cités ne rendent pas compte de la diversité des régimes (Westoby 1978), et cela même quand on tient compte des contraintes nutritionnelles déjà évoquées (Pyke et al 1977, Maizeret 1988, Owen-Smith 1993). C'est pourquoi certains auteurs remplacent aujourd'hui le critère d'optimisation par un critère moins strict de satisfaction : un aliment serait consommé quand il présente suffisamment d'intérêt et non uniquement quand c'est le meilleur (Emmans et Kyriazakis 1994). C'est également la raison pour laquelle d'autres approches ont été proposées afin d'expliquer ce qui détermine les choix alimentaires des herbivores. Concluons cependant que, même si les modèles qui s'inspirent de l'Optimal Foraging donnent parfois des résultats décevants, l'analyse des situations où les choix réalisés s'écartent de ceux annoncés ouvre d'intéressantes voies de recherches (Owen-Smith 1993).

\section{2 / La " sagesse nutritionnelle "}

De par leur importance, les contraintes nutritionnelles constituent le fondement de plusieurs théories, dans lesquelles il s'agit soit d'éviter de consommer en trop grande quantité des composés toxiques, soit d'équilibrer les éléments nutritifs indispensables à l'animal.

La grande variété de composés secondaires contenus dans les plantes et la toxicité de certains d'entre eux conduiraient les herbivores à diversifier leur régime, et à échantillonner tout en considérant avec précaution les aliments qu'ils ne connaissent pas (Freeland et Janzen 1974, Westoby 1978, Maizeret 1988). Une telle stratégie permettrait de repérer les végétaux qui peuvent être consommés, et de limiter la concentration de chaque composé secondaire ingéré. Toutes les espèces d'herbivores n'ont pas les mêmes capacités pour détoxiquer et éliminer ces composés secondaires, ce qui pourrait en partie expliquer leurs choix différents (Robbins et al 1991). 
Parce que leur régime est en général de meilleure qualité que ce qui leur est offert, les herbivores semblent doués d'une "sagesse nutritionnelle " qui leur permet de sélectionner des régimes équilibrés et suffisamment riches en nutriments indispensables (Westoby 1974, Maizeret 1988, Provenza et Balph 1990). Le fait de prendre en considération la nécessité de consommer un nutriment limitant (Owen-Smith et Novellie 1982) comme par exemple le sodium (Belovsky 1978) rend acceptable les prédictions issues de ces modèles et, récemment, Wallis de Vries et Schippers (1994) ont montré qu'ils rendaient bien mieux compte de la distribution spatiale de bovins dans un milieu très hétérogène, quand ils introduisaient dans leur modèle un objectif de maximisation de l'ingestion du sodium et du phosphore.

De telles théories impliquent que les animaux soient capables de déterminer les caractéristiques nutritionnelles et les composés toxiques des plantes qu'ils consomment. Ovins et bovins savent rectifier précisément une carence en sodium en buvant des solutions salées (Denton et Sabine 1961, Sly et Bell 1979). A l'herbe, les animaux savent utiliser les pierres à sel, mais ils ne sont pas toujours aptes à corriger dans de bonnes proportions un déficit en sodium ou en phosphore en ingérant un complément ou en choisissant les espèces qu'ils pâturent (Arnold 1985). Alors que les rongeurs préfèrent les aliments qui, nutritionnellement, leur sont le plus favorable (Provenza et Balph 1987, Cassini 1994), les herbivores semblent avoir de grandes difficultés à évaluer la composition de ce qu'ils ingèrent et à adapter leur régime en conséquence (Arnold et Hill 1972, Zahorik et Houpt 1977, Arnold 1985, Provenza et Balph 1987, 1990). Ceci est en partie lié au fait, au moins au pâturage, que les herbivores, à la différence des rongeurs omnivores, font de longs repas au cours desquels ils ingèrent différentes espèces végétales aux caractéristiques variées. Les particularités digestives des ruminants et les caractéristiques des fourrages retardent la libération de certains éléments dans le rumen, et les fermentations ruminales modifient la nature de ce qui est absorbé. L'identification d'effets individualisés des aliments est donc plus délicate. En revanche, quand les herbivores sont confrontés à des situations plus simples avec un choix limité, ils sont capables de relier l'ingestion d'un aliment à ses conséquences positives ou négatives, et ceci influence leurs préférences ultérieures. Par exemple, Thorhallsdottir (1991) et Burritt et Provenza (1992) ont offert à des moutons alternativement (un jour sur deux) deux aliments de valeurs nutritives différentes, chacun d'entre eux associé à un arôme particulier. Les animaux ont reconnu les conséquences postingestives de chaque aliment puisqu'ils ont acquis une préférence pour l'arôme associé à celui de meilleure qualité. Cette préférence persiste même quand cet arôme n'est plus associé à une quelconque caractéristique nutritionnelle. De même, chez le mouton, une perfusion d'acides gras volatiles (AGV) va, selon son importance, stimuler ou limiter l'ingestion de l'aliment qu'elle accompagne (Farningham et Whyte 1993, Provenza 1995), et influencer son acceptation ultérieure (Provenza 1995). Un excès de consommation de céréales est susceptible d'entraîner une modification des choix des ovins et des bovins en faveur d'aliments habituellement moins préférés (Provenza 1995). Plus généralement, les ruminants acquièrent des aversions alimentaires pour des aliments associés à des composés toxiques (e.g. Olsen et Ralphs 1986, Zahorik et al 1990), et limitent l'ingestion de régimes trop riches (e.g. Nicholson et al 1992) ou au contraire déficients (e.g. Egan et Rogers 1978) en énergie ou en certains nutriments. L'effet de l'ingestion de composés toxiques sur les choix est rapide puisqu'il peut être obtenu en moins d'une heure selon Provenza et al (1993). Il est persistant puisque des animaux refusent pendant plusieurs mois de consommer une espèce préalablement associée au chlorure de lithium (Burritt et Provenza 1990, Lane et al 1990). Quand ils ingèrent un composé toxique, les animaux sont capables d'un discernement qui traduit leurs possibilités de mémorisation. Par exemple, Burritt et Provenza (1991) ont montré que des moutons recevant du chlorure de lithium juste après un repas constitué de quatre aliments connus et d'un nouveau, associaient l'effet négatif $\mathrm{du} \mathrm{LiCl}$ à ce seul aliment nouveau, qu'ils rejetaient ensuite.

Les ruminants percevraient la valeur des aliments non toxiques par le biais d'indicateurs internes tels que la concentration des produits de fermentations microbiennes dans le rumen ( $\mathrm{AGV}$, ammoniaque), de certaines hormones (insuline, CCK) ou de certains métabolites ( $\mathrm{AGV}$, acides aminés) sanguins, informations relayées par la libération de neuromédiateurs (sérotonine, dopamine, CCK) au niveau du cerveau (Provenza et Balph 1990, Provenza 1995). Quand, pendant une phase d'ingestion, un herbivore consomme un seul aliment ou un mélange simple dans lequel seul un des fourrages est inconnu, il est capable d'identifier les effets positifs et surtout négatifs de son choix, de s'en souvenir et ainsi d'acquérir une certaine sagesse nutritionnelle (Provenza 1995). Au pâturage, les animaux ont peut être développé des tactiques d'échantillonnage des espèces nouvelles dont ils ignorent la qualité, qui leur permettent d'identifier les conséquences de leur ingestion, par exemple, ils pourraient ne consommer qu'une seule espèce inconnue à la fois, et en ingérer tant qu'ils ne perçoivent pas de conséquences négatives.

En fait, les herbivores associent les conséquences post-ingestives de leurs choix à des phénomènes sensoriels, ce qui leur permet ensuite de reconnaitre les aliments offerts, et de les choisir ou de les rejeter. Cependant, la reconnaissance externe des fourrages ne semble pas toujours suffisante pour que les herbivores se souviennent à long terme de
Les herbivores semblent doués d'une sagesse nutritionnelle qui leur permet de sélectionner des régimes équilibrés et suffisamment riches en nutriments indispensables. 
leurs caractéristiques nutritionnelles. Pour cela, ils doivent périodiquement consommer de petites quantités des différents fourrages (Westoby 1978, Illius et al 1992), et ceci d'autant plus que leur valeur nutritionnelle évolue rapidement (Dumont et al 1995). Cette hypothèse implique cependant qu'une fois un aliment connu, les herbivores sont capables de se remémorer rapidement les conséquences de son ingestion en en consommant très peu.

Enfin, Newman et al (1992) ont observé que des brebis maintenues depuis plus de deux mois sur des parcelles de trèfle ou de raygrass choisissent préférentiellement l'autre espèce quand on leur offre des micro-placettes de ray-grass et de trèfle. Une des hypothèses invoquées pour expliquer ce comportement est une préférence pour ce qui est rare, qui, selon ces auteurs, permettrait aux animaux de maintenir la diversité de leur flore microbienne, et par conséquent de conserver une possibilité d'adaptation à un changement brutal de régime.

\section{3 / La place de l'hédonisme}

Aux partisans des théories qui s'appuient sur les contraintes nutritionnelles que rencontrent les herbivores, s'opposent ceux qui pensent que leurs choix sont avant tout dictés par la recherche de sensations agréables, gustatives, olfactives et tactiles (Arnold 1985, Provenza et Balph 1990). Alors que l'animal utilise la vue pour s'orienter au pâturage, c'est surtout par le goût que se feraient ses choix alimentaires (Arnold 1966, Krueger et al 1974). Des variations de l'équipement sensoriel des animaux pourraient expliquer des différences de choix entre espèces et entre individus d'une même espèce (Arnold 1985). Une sensation agréable pourrait également résulter de la consommation d'un aliment nouveau (Allen 1972, Newman et al 1992), cette réaction psychologique pouvant expliquer en partie la diversité des régimes.

\section{4 / Expérience dans le jeune âge et apprentissage}

L'expérience des animaux influence également leurs choix alimentaires. Par exemple, des agneaux autorisés à pâturer avec leur mère soit du trèfle, soit du ray-grass pendant une courte période avant ou pendant le sevrage, choisissent préférentiellement par la suite l'espèce qu'ils connaissent déjà (Ramos et Tennessen 1992). De même, des brebis Caussenardes des garrigues qui exploitent ces parcours depuis leur jeune âge y consomment plus de ligneux que des brebis Mérinos récemment introduites (Thiault et al 1979). Des ovins, des bovins ou des caprins, placés dans un milieu qu'ils ne connaissent pas, pâturent jusqu'à $20 \%$ plus longtemps, mais ingèrent jusqu'à $40 \%$ moins que des animaux expérimentés (Provenza et Balph 1987). Ceci est peut être dû à leur plus faible vitesse d'ingestion sur des couverts qu'ils ne sont pas habitués à pâturer (Flores et al 1989), ou à une moins grande acceptation des espèces qui leur sont inconnues. Les herbivores ingèrent également plus de plantes toxiques dans un milieu nouveau (Provenza et Balph 1990).

Les expériences alimentaires dans le jeune âge tout comme l'apprentissage avec la mère semblent jouer un rôle primordial dans l'élaboration des préférences. Les expériences alimentaires dans le jeune âge influencent plus les choix des animaux que leurs expériences ultérieures (Arnold et Maller 1977). La période au cours de laquelle l'appareil digestif du jeune ruminant se transforme, semble particulièrement propice à une imprégnation alimentaire (Provenza et Balph 1987, Squibb et al 1990). Des agneaux nourris précocement avec un fourrage riche en fibres et de faible valeur nutritionnelle, acceptent plus volontiers une fois sevrés de consommer des fourrages grossiers, et semblent également mieux les digérer (Distel et al 1994). De même, des chèvres qui ont consommé des plantes riches en tanins dans leur jeune âge sont par la suite plus aptes à détoxiquer les produits de la dégradation de ces composés secondaires (Distel et Provenza 1991). Sur des savanes tropicales à évolution rapide, les choix alimentaires de chèvres créoles reflètent en partie la composition botanique du parcours l'année de leur naissance (Biquand et Biquand-Guyot 1992). Plusieurs espèces sont également particulièrement consommées par les individus d'une même lignée, ce qui indique une certaine transmission sociale des préférences. C'est en fait surtout la mère qui a un rôle majeur dans l'acquisition des préférences du jeune herbivore. Thorhallsdottir et al (1990) ont montré que des agneaux à qui on permettait de consommer un nouvel aliment avec leur mère en ingèrent par la suite environ deux fois plus que des agneaux qui ont eu la même expérience précoce avec une autre femelle. Ces derniers en consomment cependant encore deux fois plus que des agneaux exposés seuls à l'aliment. Enfin, Mirza et Provenza $(1990,1994)$ ont récemment observé qu'en présence de leur mère, des agneaux apprenaient rapidement à éviter un aliment toxique et à sélectionner une alternative satisfaisante.

\section{5 / Contraintes morphologiques et digestives, adaptation physiologique aux végétaux consommés}

Les caractéristiques morphologiques et physiologiques des herbivores se traduisent par des différences de besoins énergétiques, d'aptitude au tri et de capacité digestive, qui influencent largement leurs choix. La masse 
corporelle détermine la taille des compartiments digestifs et, pour une large part, la taille de la bouche de l'animal. Le volume digestif des herbivores augmente linéairement avec leur masse corporelle (Demment et Van Sœest 1985). Les espèces de petit format, qui ont des besoins énergétiques ramenés à leur volume digestif plus importants que les espèces de grand format, doivent donc sélectionner des fourrages de meilleure qualité (Demment et Van Scest 1985, Demment et Greenwood 1988). Pour une même composition du régime, les petits ruminants digèrent également moins bien les fourrages grossiers. L'écart de digestibilité entre ovins et bovins atteint en moyenne 5 points pour cent pour les fourrages pauvres (Dulphy et al 1994). Cette plus grande capacité digestive des bovins a été mise en relation avec le temps de séjour plus long des aliments dans leur tube digestif (Carle et Dulphy 1980, Poppi et al 1981), ceci se retrouvant d'une manière plus générale entre espèces de différents formats (Demment et Greenwood 1988). En revanche, les espèces de grand format sont moins aptes à trier. Elles sont particulièrement handicapées sur les couverts hétérogènes où les éléments de qualité sont rares ou moins accessibles (e.g. Zeeman et al 1983, Hodgson et al 1991). Elles sont également désavantagées sur les couverts herbacés ras où la profondeur des prises alimentaires est limitée (Illius et Gordon 1987). La taille des prises est alors surtout déterminée par la largeur de la barre incisive, qui augmente avec la masse corporelle plus lentement (autour de PV ${ }^{0,35}$ ) que les besoins énergétiques (Clutton-Brock et Harvey 1983, Gordon et Illius 1988). Conséquence de cela, les bovins choisissent plus systématiquement que les ovins de pâturer les placettes d'herbe végétative les plus hautes (Illius et Gordon 1990). Cependant, la plus grande aptitude des bovins comparés aux ovins à exploiter des fourrages grossiers n'est pas uniquement la conséquence de leur plus faible aptitude au tri et de leurs plus grandes capacités ingestive et digestive. Elle résulte aussi en partie de différences inter-spécifiques de préférences, les bovins choisissant plus volontiers que les ovins de consommer un fourrage grossier associé à un fourrage de meilleure qualité, dans des situations où les aptitudes des animaux n'influencent pas leurs choix (Dumont et al 1995, Dumont et Petit 1995).

Le type de digestion (ruminale ou caecale) joue à la fois sur la capacité de digestion et d'ingestion des herbivores (Janis 1976, Hanley 1982, Duncan et al 1990). Chez les ruminants, les particules doivent être réduites à une taille suffisamment petite pour quitter le rumen, ce qui est généralement considéré comme étant susceptible de limiter les quantités ingérées. En revanche, les ruminants digèrent mieux les parois végétales (Dulphy et al 1994) et n'auraient par conséquent pas besoin d'ingérer, à masse corporelle égale, les mêmes quantités que les herbivores à digestion caecale. Malgré cela, des bovins et des chevaux à l'entretien ou en croissance ingèrent à l'auge des quantités voisines de matière sèche par $\mathrm{kg}$ de poids vif (Chenost et Martin-Rosset 1985, Cymbaluk 1990), même si une augmentation de la teneur en fibres des fourrages affecte moins l'ingestion chez les chevaux.

Les choix alimentaires des ruminants ont enfin été expliqués selon une théorie d'adaptation éco-physiologique des différentes espèces aux organes végétaux qu'elles consomment. L'importance du réticulo-rumen par rapport à la masse corporelle, mais également d'autres caractéristiques anatomiques et physiologiques de leur appareil digestif (importance des glandes salivaires et des villosités de la paroi dorsale du rumen, développement de la muqueuse de l'abomasum, longueur de l'intestin,...) ont permis à Hofmann $(1973,1989)$ de classer les ruminants en trois grandes catégories: les "grazers" (ou grass and roughage eaters : bovins, ovins), les "browsers" (ou concentrate selectors : chevreuil) et les consommateurs mixtes (cerf, caprins). Les "grazers », grâce à leur énorme réticulorumen rapporté à leur poids, digèrent très efficacement les parois végétales, alors que les "browsers" possèdent un ensemble de structures et de sites de digestion qui leur permettent d'exploiter au mieux la gamme des dicotylédones. Par ailleurs, rappelons que le régime qu'ils sélectionnent détermine largement l'organisation sociale des ruminants africains (Jarman 1974). Les espèces sélectives vivent plutôt en couple ou en petits groupes, alors que les « grazers " forment souvent de grands troupeaux.

\section{Conclusion}

Il existe donc plusieurs théories qui tentent d'expliquer les principes de la sélection alimentaire des herbivores au pâturage. Selon les conditions dans lesquelles les animaux se trouvent, certains facteurs auront plus ou moins d'importance. Par exemple, la nécessité d'éviter de consommer des composés secondaires toxiques n'influence certainement pas les choix des animaux pâturant des prairies tempérées. Il semble clair que les caractéristiques morpho-physiologiques des herbivores définissent les grandes lignes de leurs stratégies alimentaires et expliquent les différences entre espèces. En revanche, au sein de chaque espèce les expériences alimentaires dans le jeune âge, l'apprentissage avec la mère et la reconnaissance des conséquences post-ingestives des choix réalisés jouent un rôle dans l'acquisition d'habitudes alimentaires et expliquent à la fois une certaine «sagesse nutritionnelle " et la plasticité des comportements observés. Remarquons enfin que l'état physiologique des animaux module l'expression de leurs préférences et de leurs aptitudes. En fonction de la quantité de matière sèche qu'ils doivent ingérer pour couvrir leurs besoins énergétiques, les herbivores auront plus ou moins de temps pour rechercher leurs espèces préférées. Ceci se répercutera sur la composition de leur ration journalière.
Les

caractéristiques

morphologiques et physiologiques des herbivores

expliquent

largement les

différences de

choix alimentaires

entre espèces. 


\section{Références bibliographiques}

Allen J.A., 1972. Evidence for stabilizing and apostatic selection by wild blackbirds. Nature, 237, 348349 .

Arnold G.W., 1966. The special senses in grazing animals. II. Smell, taste, and touch and dietary habits in sheep. Aust. J. Agric. Res., 17, 531-542.

Arnold G.W., 1985. Ingestive behaviour. In : Ethology of farm animals. A comprehensive study of the behavioural features of the common farm animals, A.F. Fraser (ed), 183-200. Elsevier, Amsterdam.

Arnold G.W., 1987. Influence of the biomass, botanical composition and sward height of annual pastures on foraging behaviour by sheep. J. Appl. Ecol., $24,759-772$.

Arnold G.W., Hill J.L., 1972. Chemical factors affecting selection of food plants by ruminants. In : Phytochemical ecology, J.B. Harborne (ed), 71-101. Academic Press, London.

Arnold G.W., Maller R.A., 1977. Effects of nutritional experience in early and adult life on the performance and dietary habits of sheep. Appl. Anim. Ethol., 3, 5-26.

Belovsky G.E., 1978. Diet optimization in a generalist herbivore: the moose. Theor. Popul. Biol., 14, 105-134.

Belovsky G.E., 1986. Optimal foraging and community structure : implications for a guild of generalist grassland herbivores. Oecologia, 70, 35-52.

Biquand S., Biquand-Guyot V., 1992. The influence of peers, lineage and environment on food selection of the criollo goat (Capra hircus). Appl. Anim. Behav. Sci., 34, 231-245.

Broom D.M., Arnold G.W., 1986. Selection by grazing sheep of pasture plants at low herbage availability and responses of the plants to grazing. Aust. J. Agric. Res., 37, 527-538.

Burritt E.A., Provenza F.D., 1990. Food aversion learning in sheep : persistence of conditioned taste aversions to palatable shrubs (Cercocarpus montanus and Amelanchier alnifolia). J. Anim. Sci., 68, 1003-1007.

Burritt E.A., Provenza F.D., 1991. Ability of lambs to learn with a delay between food ingestion and consequences given meals containing novel and familiar foods. Appl. Anim. Behav. Sci., 32, 179-189.

Burritt E.A., Provenza F.D., 1992. Lambs form preferences for non nutritive flavors paired with glucose. J. Anim. Sci., 70, 1133-1136.

Carle B., Dulphy J.P., 1980. Comportement alimentaire comparé des ovins et des bovins. Relation avec la digestion des aliments. Reprod. Nutr. Dévelop., $20,1633-1639$.

Cassini M.H., 1994. Behavioral mechanisms of selection of diet components and their ecological implications in herbivorous mammals. J. Mammal., $75,733-740$.

Chenost M., Martin-Rosset W., 1985. Comparaison entre espèces (mouton, cheval, bovin) de la digestibilité et des quantités ingérées des fourrages verts. Ann. Zootech., 34, 291-312.
Clutton-Brock T.H., Harvey P.H., 1983. The functional significance of variation in body size among mammals. Spec. Publ. Amer. Soc. Mamm., 7, 632-663.

Cymbaluk N.F., 1990. Comparison of forage digestion by cattle and horses. Can. J. Anim. Sci., 70, 601-610.

Demment M.W., Greenwood G.B., 1988. Forage ingestion : effects of sward characteristics and body size. J. Anim. Sci., 66, 2380-2392.

Demment M.W., Van Sœst P.J., 1985. A nutritional explanation for body-size patterns of ruminant and non ruminant herbivores. Am. Nat., 125, 641-672.

Denton D.A., Sabine J.R., 1961. Selective appetite for $\mathrm{Na}^{+}$shown by $\mathrm{Na}^{+}$deficient sheep. J. Physiol., 157, $97-116$.

Distel R.A., Provenza F.D., 1991. Experience early in life affects voluntary intake of Blackbrush by goats. J. Chem. Ecol., 17, 431-450.

Distel R.A., Villalba J.J., Laborde H.E., 1994. Effects of early experience on voluntary intake of low-quality roughage by sheep. J. Anim. Sci., 72, 1191-1195.

Dulphy J.P., Jouany J.P., Martin-Rosset W., Thériez M., 1994. Aptitudes comparées de différentes espèces d'herbivores domestiques à ingérer et digérer des fourrages distribués à l'auge. Ann. Zootech., $43,11-32$.

Dumont, B., Petit M., 1995. An indoor method for studying sheep's and cattle's preferences at pasture. Appl. Anim. Behav. Sci. (sous presse).

Dumont B., Petit M., D'hour P., 1995. Choice of sheep and cattle between vegetative and reproductive cocksfoot patches. Appl. Anim. Behav. Sci., 43, 1-15.

Duncan P., Foose T.J., Gordon I.J., Gakuhu C.G., Lloyd M., 1990. Comparative nutrient extraction from forages by grazing bovids and equids : a test of the nutritional model of equid/bovid competition and coexistence. Oecologia, 84, 411-418.

Egan A.R., Rogers Q.R., 1978. Amino acid imbalance in ruminant lambs. Aust. J. Agric. Res., 29, 12631279 .

Emmans G.C., Kyriazakis, I., 1994. What criteria do animals use when making feeding decisions? In : $45^{\text {th }}$ Annual Meeting of the European Association for Animal Production, Edinburgh, 5-8 September 1994, Abstract N2.4, 126

Farningham D.A.H., Whyte C.C., 1993. The role of propionate and acetate in the control of food intake in sheep. Br. J. Nutr., 70, 37-46.

Flores E.R., Provenza F.D., Balph D.F., 1989. The effect of experience on the foraging skill of lambs : importance of plant form. Appl. Anim. Behav. Sci., $23,285-291$.

Freeland W.J., Janzen D.H., 1974. Strategies in herbivory by mammals : the role of plant secondary compounds. Am. Nat., 108, 269-289.

Gordon I.J., Illius A.W., 1988. Incisor arcade structure and diet selection in ruminants. Funct. Ecol., 2, 15-22. 
Hanley T.A., 1982. The nutritional basis for food selection by ungulates. J. Range Manage., 35, 146151.

Hodgson J., Forbes T.D.A., Armstrong R.H., Beattie M.M., Hunter E.A., 1991. Comparative studies of the ingestive behaviour and herbage intake of sheep and cattle grazing indigenous hill plant communities. J. Appl. Ecol., 28, 205-227.

Hofmann R.R., 1973. The ruminant stomach (stomach structure and feeding habits of East African game ruminants). Vol.2 East Afr. Monogr. Biol., E.A. Lit. Bureau, Nairobi, 364 pp.

Hofmann R.R., 1989. Evolutionary steps of ecophysiological adaptation and diversification of ruminants : a comparative view of their digestive system. Oecologia, 78, 443-457.

Illius A.W., Gordon I.J., 1987. The allometry of food intake in grazing ruminants. J. Anim. Ecol., 56, 989-999.

Illius A.W., Gordon I.J., 1990. Constraints on diet selection and foraging behaviour in mammalian herbivores. In : Behavioural mechanisms of food selection, R.N. Hugues (ed), 369-393. Springer-Verlag, Berlin.

Illius A.W., Clark D.A., Hodgson J., 1992. Discrimination and patch choice by sheep grazing grass-clover swards. J. Anim. Ecol., 61, 183-194.

Janis C., 1976. The evolutionary strategy of the Equidae and the origins of rumen and cecal digestion. Evolution, 30, 757-774.

Jarman P.J., 1974. The social organisation of antelope in relation to their ecology. Behaviour, 48, 215267.

Kenney P.A., Black J.L., 1984. Factors affecting diet selection by sheep. I. Potential intake rate and acceptability of feed. Aust. J. Agric. Res., 35, 551563.

Krueger W.C., Laycock W.A., Price D.A., 1974. Relationships of taste, smell, sight, and touch to forage selection. J. Range Manage., 27, 258-262.

Lane M.A., Ralphs M.H., Olsen J.D., Provenza F.D., Pfister J.A., 1990. Conditioned taste aversion potential for reducing cattle loss to larkspur. J. Range Manage., 43, 127-131.

Maizeret C., 1988. Stratégies alimentaires des chevreuils : les fondements écologiques d'une diversification du régime. Acta OEcologica OEcol. Applic., 9, 191-211.

Mirza S.N., Provenza F.D., 1990. Preference of the mother affects selection and avoidance of foods by lambs differing in age. Appl. Anim. Behav. Sci., 28, 255-263.

Mirza S.N., Provenza F.D., 1994. Socially induced food avoidance in lambs : direct or indirect marternal influence? J. Anim. Sci., 72, 899-902.

Newman J.A., Parsons A.J., Harvey A., 1992. Not all sheep prefer clover : diet selection revisited. J. Agric. Sci. (Camb.), 119, 275-283.

Newman J.A., Parsons A.J., Thornley J.H.M., Penning P.D., Krebs J.R., 1995. Optimal diet selection by a generalist grazing herbivore. Funct. Ecol., 9, 255-268.
Nicholson J.W.G., Charmley E., Bush R.S., 1992. The effect of supplemental protein source on ammonia levels in rumen fluid and blood and intake of alfalfa silage by beef cattle. Can. J. Anim. Sci., 72, 853-862.

Olsen J.D., Ralphs M.H., 1986. Feed aversion induced by intraruminal infusion with larkspur extract in cattle. Am. J. Vet. Res., 47, 1829-1833.

Owen-Smith N., 1993. Evaluating optimal diet models for an african browsing ruminant, the kudu : how constraining are the assumed constraints? Evol. Ecol., 7, 499-524.

Owen-Smith N., Novellie P., 1982. What should a clever ungulate eat? Am. Nat., 119, 151-178.

Parsons A.J., Newman J.A., Penning P.D., Harvey A., Orr R.J., 1994. Diet preference of sheep : effects of recent diet, physiological state and species abundance. J. Anim. Ecol., 63, 465-478.

Penning P.D., Rook A.J., Orr R.J., 1991. Patterns of ingestive behaviour of sheep continuously stocked on monocultures of ryegrass or white clover. Appl. Anim. Behav. Sci., 31, 237-250.

Poppi D.P., Minson D.J., Ternouth J.H., 1981. Studies of cattle and sheep eating leaf and stem fractions of grasses. I. The voluntary intake, digestibility and retention time in the reticulo-rumen. Aust. J. Agric. Res., 32, 99-108.

Provenza F.D., 1995. Postingestive feedback as an elementary determinant of food preference and intake in ruminants. J. Range Manage., 48, 2-17.

Provenza F.D., Balph D.F., 1987. Diet learning by domestic ruminants : theory, evidence and practical implications. Appl. Anim. Behav. Sci., 18, 211-232.

Provenza F.D., Balph D.F., 1990. Applicability of five diet-selection models to various foraging challenges ruminants encounter. In : Behavioural mechanisms of food selection, R.N. Hugues (ed), 423-460. Springer-Verlag, Berlin.

Provenza F.D., Nolan J.V., Lynch J.J., 1993. Temporal contiguity between food ingestion and toxicosis affects the acquisition of food aversions in sheep. Appl. Anim. Behav. Sci., 38, 269-281.

Pyke G.H., Pulliam H.R., Charnov E.L., 1977. Optimal foraging : a selective review of theories and tests. Quart. Rev. Biol., 52, 137-153.

Ramos A., Tennessen T., 1992. Effect of previous grazing experience on the grazing behaviour of lambs. Appl. Anim. Behav. Sci., 33, 43-52.

Robbins C.T., Hagerman A.E., Austin P.J., McArthur C., Hanley T.A., 1991. Variation in mammalian physiological responses to a condensed tannin and its ecological implications. J. Mammal., 72, 480-486.

Schœener T.W., 1971. Theory of feeding strategies Annu. Rev. Ecol. Syst., 2, 369-404.

Sly J., Bell F.R., 1979. Experimental analysis of the seeking behaviour observed in ruminants when they are sodium deficient. Physiol. Behav., 22, 499-505.

Squibb R.C., Provenza F.D., Balph D.F., 1990. Effect of age of exposure on consumption of a shrub by sheep. J. Anim. Sci., 68, 987-997.

Stephens D.W., Krebs J.R., 1986. Foraging theory. Princeton Univ. Press, Princeton, NJ, 247 pp. 
Thiault M., Prud'hon M., Reboul G., Béchet G., Molénat G., Thériez M., 1979. Amélioration pastorale de la garrigue. In: Utilisation par les ruminants des pâturages d'altitude et parcours méditerranéens, G. Molénat et $\mathrm{R}$. Jarrige (eds), 375-396. INRA Publications, Versailles.

Thorhallsdottir A.G., 1991. Factors determining diet selection by sheep. In : IV th International Rangeland Congress, P. Daget et M. Kernick (eds), Abstr. 297'. CIHEAM/IAM, Montpellier.

Thorhallsdottir A.G., Provenza F.D., Balph D.F., 1990. Ability of lambs to learn about novel foods while observing or participating with social models. Appl. Anim. Behav. Sci., 25, 25-33.

Verlinden C., Wiley R.H., 1989. The constraints of digestive rate : an alternative model of diet selection. Evol. Ecol., 3, 264-273.

Wallis de Vries M.F., Schippers P., 1994. Foraging in a landscape mosaic : selection for energy and minerals in free-ranging cattle. Oecologia, 100, 107-117.
Westoby M., 1974. An analysis of diet selection by large generalist herbivores. Am. Nat., 108, 290-304.

Westoby M., 1978. What are the biological bases of varied diets? Am. Nat., 112, 627-631.

Zahorik D.M., Houpt K.A., 1977. The concept of nutritional wisdom : applicability of laboratory learning models to large herbivores. In : Learning mechanisms in food selection, L.M. Barker et al (eds), 45-70. Baylor Univ. Press, Waco, Texas.

Zahorik D.M., Houpt K.A., Swartzman-Andert J., 1990. Taste-aversion learning in three species of ruminants. Appl. Anim. Behav. Sci., 26, 27-39.

Zeeman P.J.L., Marais P.G., Cotsee M.J., 1983. Nutrient selection by cattle, goats and sheep on natural Karoo pasture. 1. Digestibility of organic matter. S. Afr. J. Anim. Sci., 13, 236-239.

\begin{abstract}
Dietary choice determinism for grazing herbivores : principal theories.

The objective of maintaining adequate grassland requires a better understanding of the conditions affecting the dietary choices of herbivores. The studies in this area have long been based on the Optimal Foraging Theory, which states that an animal's feeding decisions are dictated by its will to maximize its energy balance. In order to better explain the variety of observed diets, however, other approaches have been suggested. These are largely inspired by the nutritional constraints of
\end{abstract}

Abstract herbivores. The morpho-physiological characteristics of the animals largely explain the different choices between species. In each species there is evidence that learning early in life, learning from mother, and learning the consequences of nutritional choices from postingestive feedback all play a role in the acquisition of eating habits and explain both a certain nutritional wisdom and the flexibility in the observed behaviour.

DUMONT B., 1995. Déterminisme des choix alimentaires des herbivores au pâturage: principales théories. INRA Prod. Anim., 8 (4), 285-292. 